\title{
LINKS BETWEEN SYMBIOTIC AND PLANETARY NEBULAE
}

\author{
J. A. López, ${ }^{1}$ K. Escalante, ${ }^{1}$ and H. Riesgo-Tirado ${ }^{1}$
}

Planetary nebulae (PNe) represent a well defined stage of stellar evolution, where the characteristics of both, the central star and the gaseous envelope have defined properties. A large fraction of PNe contain binary nuclei, therefore, binary evolution plays an important role in the AGB and protoplanetary stages under certain circumstances. Symbiotic nebulae with extended envelopes (D-type), are identified with binary systems where the primary component is usually a Mira giant and the companion a hot white dwarf. Some of these systems resemble planetary nebulae and as a consequence can lead to misleading interpretations. Here we discuss some relevant links and differences between these two types of nebulae

The planetary nebulae stage occurs near the end of the evolution of intermediate an low mass stars ( $1-9 \mathrm{M}_{\odot}$ ), right after the heavy mass-loss episodes at the asymptotic giant branch. After losing most of its envelope at the $\mathrm{AGB}$, the $\mathrm{C}-\mathrm{O}$ stellar core, now an incipient white dwarf of $\mathrm{M} \sim 0.55 \mathrm{M}_{\odot}$ and $\mathrm{R}$ $\sim 0.4 \mathrm{R}_{\odot}$, gradually increases its effective temperature until it produces enough Lyman photons to ionize the surrounding material previously shed at the AGB. The emission line spectrum of these nebulae is characterized in the optical range by a weak atomic recombination continuum, electron densities of $\sim 10^{3} \mathrm{~cm}^{-3}$ and electron temperatures $\sim 8 \times 10^{3}$ $10^{4} \mathrm{~K}$. Conspicuous forbidden lines of oxygen, nitrogen and sulfur and Balmer recombination lines dominate the emission line spectrum. During its evolution the nebular shell develops a rich variety of morphologies which have been explained in terms of the formation of equatorial to polar density enhancements in the early stages of evolution. Progenitor masses, hydrodynamics, magnetic fields, stellar rotation and the influence of binary cores all play a role in the evolution of the nebula.

At a recent conference on Symbiotic stars (Symbiotic stars, probing stellar evolution, La Palma, Canary Islands, Spain, May 2002, eds. R.Corradi \& J. Mikolajewska, in press) a discussion on similari-

\footnotetext{
${ }^{1}$ Instituto de Astronomía, UNAM, Apdo Postal 877, 22800 Ensenada, Baja California, México
}

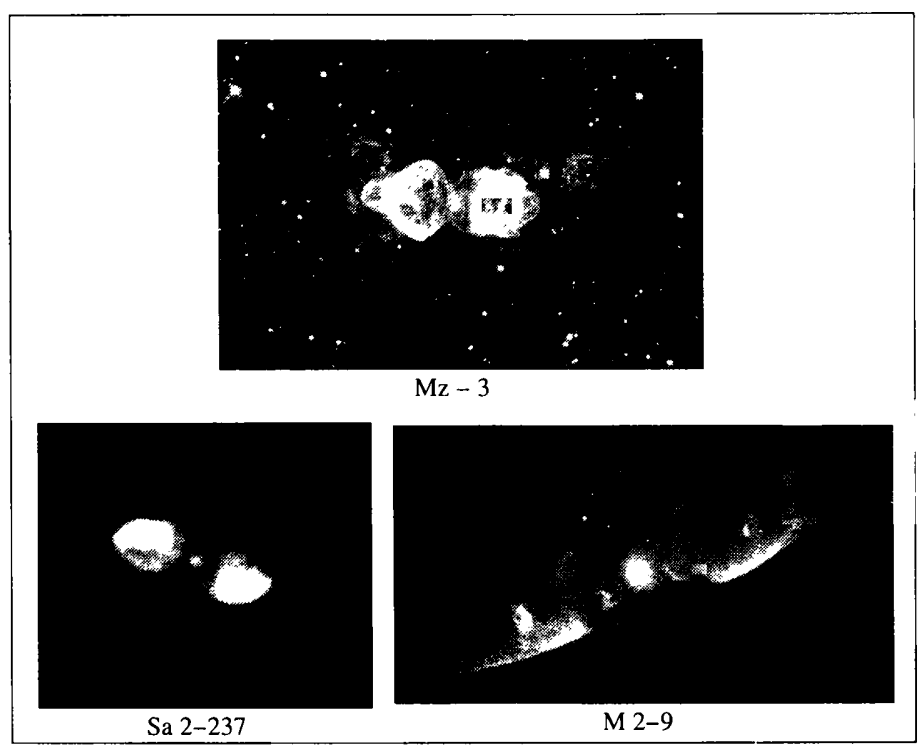

Fig. 1. Examples of symbiotic nebulae that have been classified as PNe. Mz-3 image from $H S T$, credits: R. Sahai et al. Sa 2-237 image from SPM, credits: J. A. López. M 2-9 image from HST, credits: B. Balick.

ties and differences between planetary and symbiotic nebulae took place. More recently, at the Asymmetrical Planetary Nebulae III conference, (July 2003, Mt Rainier, Washington, USA, eds. N. Soker, M. Meixener \& J. Kastner, in press) the debate arouse again. Clearly the issue is topical. The reason for this most likely is the increasing need and relevance of considering the influence of binary nuclei in the evolution of $\mathrm{PNe}$, an evolutionary stage that has mostly been modeled and understood in the past in terms of single stars; although binary cores have indeed been invoked before to explain axisymmetric morphologies (e.g. Morris 1987) and other phenomena such as common envelope evolution (e.g. Iben, 1991). However, it has been the discovery of collimated outflows and point-symmetric structures in PNe (e.g. López 1993, 2000) that revitalized binary theories for PNe (e.g. Soker \& Livio 1994). Furthermore, Soker (2000) has argued that all PN morphologies and other dynamic phenomena can be explained in terms of their particular type of binary core.

Symbiotic nebulae with extended envelopes are formed by the intense mass-loss of a red giant in the early AGB phase, usually a Mira or a slightly hotter star and an evolved, high temperature, low luminosity. white dwarf that provides the Lyman flux to ion- 


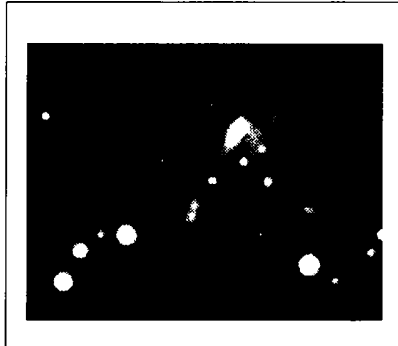

K I-2

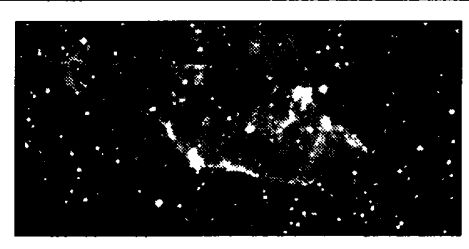

$\mathrm{KjPn} 8$

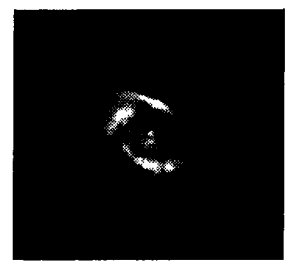

Fig. 2. Examples of planetary nebulae with a binary core. KjPn 8 images from SPM and HST, credits: J. A. López. K 1-2, credits: R. Corradi.

ize the nebula. Periods of the order of $\geq 10$ years are common in these systems (e.g. Mikolajewska, 2000). Tidal forces and wind accretion effects in this sort of binary cores provide a natural path to produce axisymmetric nebular structures (e.g. Corradi et al. 2000). Electron densities in these cases are higher than in $\mathrm{PNe}$, usuaily $\geq 10^{4} \mathrm{~cm}^{-3}$, also these nebulae have higher excitation conditions, showing strong $[O$ III] and He II emission lines. The underlying photspheric spectrum of the core is dominated by the red continuum of a F - M giant.

Therefore both, planetary and symbiotic nebulae can share the following properties: binary cores, axisymmetric morphologies, the presence of collimated, bipolar outflows and emission line spectra. Examples of PNe with binary nuclei and symbiotic nebulae that have been misidentified as PN are shown in Figure 1. Nevertheless, it must be stressed that the $P N$ phase referes to a well defined stage of evolution. that of a star that ionizes the material that it has expelled at the previous AGB stage. This star may or may not have a companion and in the latter case the companion my influence the mass-loss process and resultant geometry of the outflowing material. On the other hand for symbiotic nebula, the masslosing Mira, or red giant component has not reached the PN phase yet, whereas the WD companion has already long experienced it. Thus, in this case we have a binary where a component can be regarded as a pre-PN while the other is a post-PN. Of course, different combinations of mass ratios for the binary system together with their own rates of stellar evolution will produce akin systems, such as novae and cataclysmic variables.

\section{REFERENCES}

Corradi, R. L. M., Livio, M., Schwarz, H. E. \& Munari, U. 2000, in Asymmetrical Planetary Nebulae II, ASP Conf. Series 199, 175

Iben, I. 1991, ApJS, 76, 55

López, J. A., Meaburn, J. \& Palmer J. W. 1993, Аp.J. 415, L 135

López, J. A. 2000, RevMexAA(SC), 9, 201

Mikolajewska, J. 2000, in Asymmetrical Planetary Vebulae II, ASP Conf. Series 199, 431

Morris, M. 1987, PASP, 99, 1115

Soker, N. \& Livio, M. 1994, ApJ, 421, 219

Soker, N. 2000, MNRAS, 330, 481 